\title{
Poly- and mono(ADP-ribosyl)ation of DNA strand breaks by PARP2 and PARP3 enzymes
}

\author{
G. Zarkovic ${ }^{1}$, E.A. Belousova², I. Talhaoui ${ }^{1}$, C. Saint-Pierre ${ }^{3}$, M.M. Kutuzov², \\ B.T. Matkarimov ${ }^{4}$, D. Gasparutto ${ }^{3}$, O.I. Lavrik ${ }^{2}$, A.A. Ishchenko ${ }^{1 *}$ \\ ${ }^{1}$ Groupe «Réparation de l'ADN», Equipe Labellisée par la Ligue Nationale Contre le Cancer, \\ CNRS UMR8200, Univ. Paris-Sud, Université Paris-Saclay, Villejuif, France \\ ${ }^{2}$ Institute of Chemical Biology and Fundamental Medicine, Novosibirsk, Russia \\ ${ }^{3}$ Université Grenoble Alpes, INAC/SPrAM UMR5819 CEA CNRS UGA, Grenoble, France \\ ${ }^{4}$ National Laboratory Astana, Nazarbayev University, Astana, Kazakhstan \\ *e-mail: Alexander.ISHCHENKO@gustaveroussy.fr
}

Key words: PARP2, PARP3, DNA PARylation, BER

DNA dependent poly(ADP-ribose) polymerases (PARP1-3) play important roles in the repair of DNA strand breaks and are known to be activated in the presence of DNA strand breaks to catalyze poly- or mono(ADP-ribosyl)ation (PARylation or MARylation, respectively) of themselves or other nuclear acceptor proteins. Each PARP recognizes distinct sets of DNA structures with breaks, suggesting that PARP1, 2 and 3 have nonoverlapping functions in DNA repair. At present, the detailed molecular mechanisms of PARP-dependent DNA breaks repair remain unknown. Previously, we showed that mammalian PARP1 and PARP2 can catalyze covalent addition of ADP-ribose units not only to proteins but also to DNA strand breaks containing terminal phosphates or a 2'-OH group, thus producing a covalent PAR-DNA adduct in vitro. The PARP-catalyzed DNA PARylation is a reversible process because PAR can be entirely degraded by poly(ADP-ribose) glycohydrolase (PARG). Here, we examined DNA ADP-ribosylation activity and the DNA substrate preference of PARP3 as compared with structurally similar PARP2. PARP3 can effectively produce MAR-DNA adducts covalently linked to terminal phosphates at DSB and SSB termini of short and long DNA molecules, exhibiting similar substrate specificity with PARP2. Notably, ADP-ribosylation of 5'-terminal thiophosphates at DSB termini by PARPs generates MAR-DNA adducts resistant to PARG hydrolysis. We found that depending on configuration of DNA strand breaks, the DNA termini can become preferred acceptor sites for ADP-ribosylation as compared to proteins. According to the data obtained, we propose a putative mechanistic model of DNA strand break-oriented DNA ADP-ribosylation by PARP3 or PARP2. Our findings reveal effective PARP3- or PARP2-catalyzed ADP-ribosylation of 3-kb DNA plasmid-based substrates and DNA PARylation activity in nuclear extracts from HeLa cells. Finally, immunoblotting of purified genomic DNA from PARG-depleted HeLa cells after genotoxic treatment provides indirect evidence of the presence of PAR-DNA adducts in live cells. These results suggest that certain types of complex DNA breaks can be effectively ADP-ribosylated by PARPs in cellular response to DNA damage.

Acknowledgements: This work was supported by grants from La Ligue Nationale Contre le Cancer "Equipe LNCC 2016", EDF RB 2016-17, CNRS PRC-Russie 1074 "REDOBER”, Russian Science Foundation 14-24-0038 and 17-74-20075. 